\title{
Congenital Heminasal Hypoplasia with an Intranasal Cyst and Its Management by Subnasal Rotation Flap
}

${ }^{1}$ Chidananda R Devasamudra, ${ }^{2}$ Niveditha J Sagar, ${ }^{3}$ Rijo M Jayaraju

\begin{abstract}
Various congenital nasal deformities can occur because of the complexities of the developmental process. These conditions range from partial deformities of the nose (such as isolated absence of the nasal bones, absence of columella, absence of the septal cartilage, and absence of vomeral bone) to complete absence of the nose. Congenital heminasal hypoplasia is an extremely rare defect of embryogenesis. We present a case of 25-year-old lady with congenital heminasal hypoplasia associated with intranasal cyst. Computed tomography (CT) scan revealed gross septal deviation to left, rudiment left lateral wall of the nose, rudiment left external nasal framework, and intranasal cyst. Functional septorhinoplasty was done with subnasal rotation flap technique to gain airway on both sides of the nose.
\end{abstract}

Keywords: Heminasal hypoplasia, Intranasal cyst, Septorhinoplasty, Subnasal rotation flap.

How to cite this article: Devasamudra CR, Sagar NJ, Jayaraju RM. Congenital Heminasal Hypoplasia with an Intranasal Cyst and Its Management by Subnasal Rotation Flap. Int J Otorhinolaryngol Clin 2016;8(2):56-59.

Source of support: Nil

Conflict of interest: None

\section{INTRODUCTION}

Congenital heminasal hypoplasia is an extremely rare defect of embryogenesis. Heminasal hypoplasia is a relatively uncommon deformity with significant esthetic, functional, and social effects on the patient. ${ }^{1}$ Impaired nasal breathing due to iatrogenic or congenital narrowing of the nasal vestibule is a difficult problem, and in most cases need surgical treatment. A thorough analysis of the pathological, anatomical findings causing nasal vestibular malformation and impaired nasal breathing is a prerequisite for successful surgical treatment. ${ }^{2}$

This case report is of a 25 -year-old lady with congenital asymmetrical nostril. Computed tomography (CT) scan revealed gross septal deviation to left, rudiment

${ }^{1,2}$ Assistant Professor, ${ }^{3}$ Senior Resident

${ }^{1-3}$ Department of ENT and Facial Plastic Surgery, Sapthagiri Institute of Medical Sciences and Research Centre, Bengaluru Karnataka, India

Corresponding Author: Chidananda R Devasamudra, Assistant Professor, Department of ENT and Facial Plastic Surgery Sapthagiri Institute of Medical Sciences and Research Centre Bengaluru, Karnataka, India, Phone: +918971611749, e-mail: devasamudra@gmail.com left lateral wall of the nose, rudiment left external nasal framework, and intranasal cyst. Functional septorhinoplasty ${ }^{3}$ is done with subnasal rotation flap ${ }^{4}$ technique to gain airway on both sides of the nose.

\section{CASE REPORT}

A 25-year-old lady presented to the outpatient department with complaints of asymmetrical nostrils and unilateral (left side) nasal obstruction since birth. She did not have any other remarkable complaints during early childhood or adolescence. Endoscopic examination could not be done on left side because of narrow vestibular passage and right side was normal (Figs 1 and 2). The

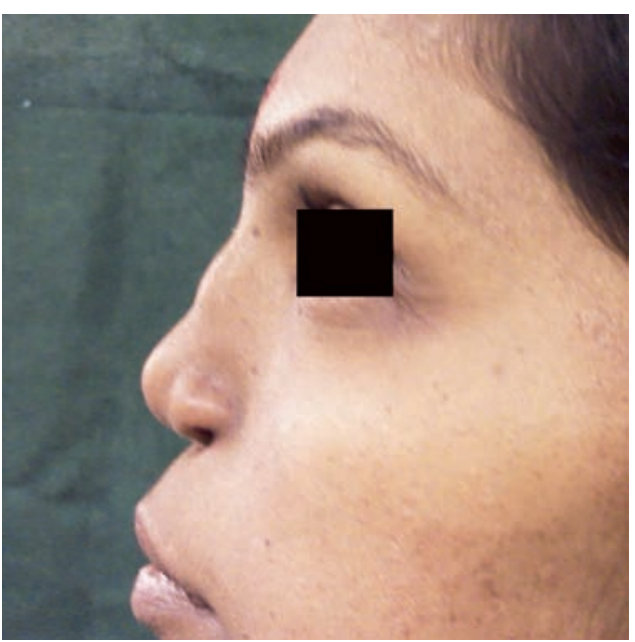

Fig. 1: The preoperative pictures of a 25-year-old lady. Note the vestibular stenosis on the left side

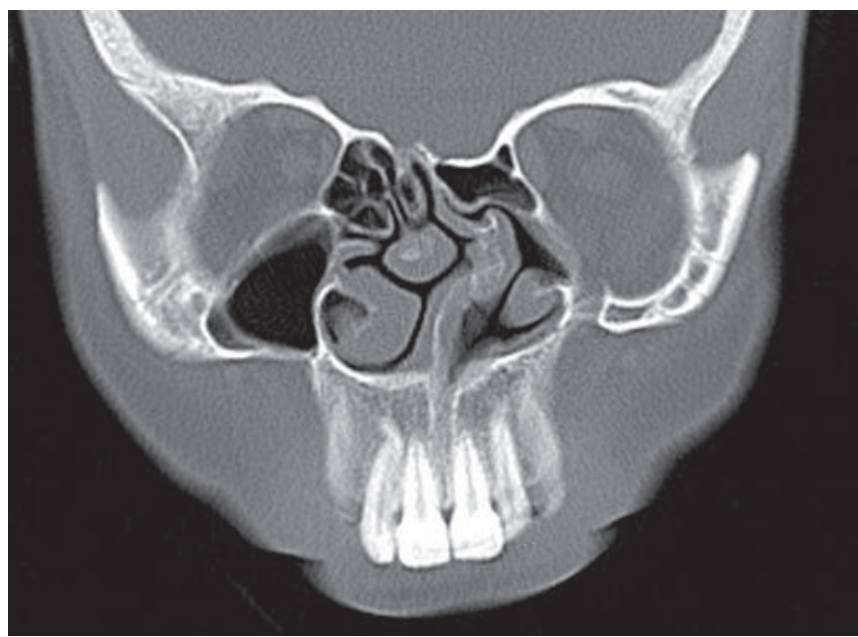

Fig. 2: Lateral view showing pseudo supratip depression due to midline intranasal cyst 


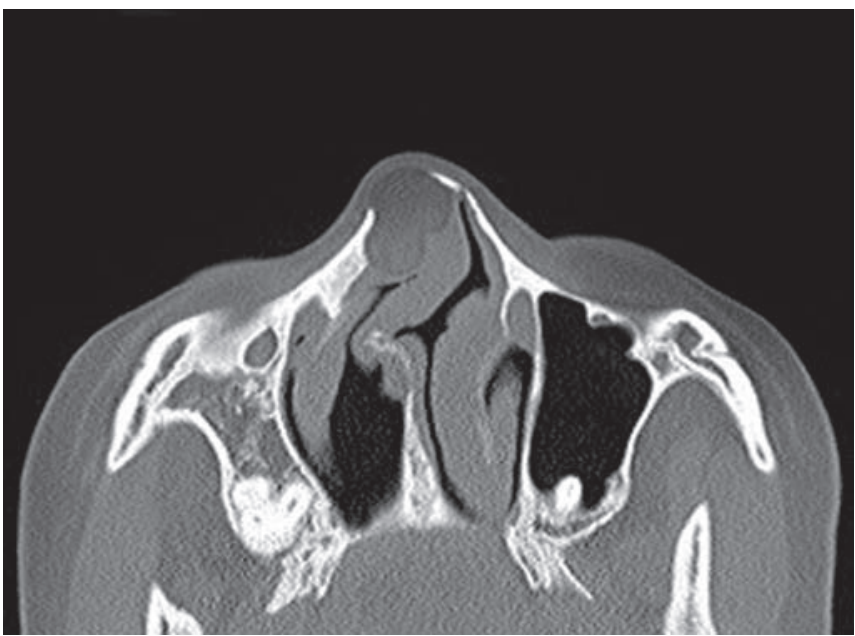

Fig. 3: ACT scan showing rudiment external nasal framework (both bony and cartilaginous) on left side, gross septal deviation to left, absent left lateral wall of the nose

patient was subjected to CT scan of nose and paranasal sinuses in order to know more about the deformities and to rule out central nervous system (CNS) involvement. It revealed left-side nasal vestibular stenosis, rudiment external nasal framework (both bony and cartilaginous) on left side, gross septal deviation to left, absent left lateral wall of the nose, underdeveloped left maxillary sinus, and cystic swelling over dorsum of the nose (Figs 3 to 5). She did not have any other congenital abnormalities. However, she had not visited any doctor previously.

\section{OPERATIVE PROCEDURE}

\section{Subnasal Rotation Flap ${ }^{4}$}

The patient was operated under general anesthesia. Oral intubation is done with South pole right angle endotracheal (RAE) tube. Local anesthesia (1\% lignocaine +1 in 100,000 adrenaline) infiltrated along the incision sites, nasal septum, and skin over the dorsum of the nose.

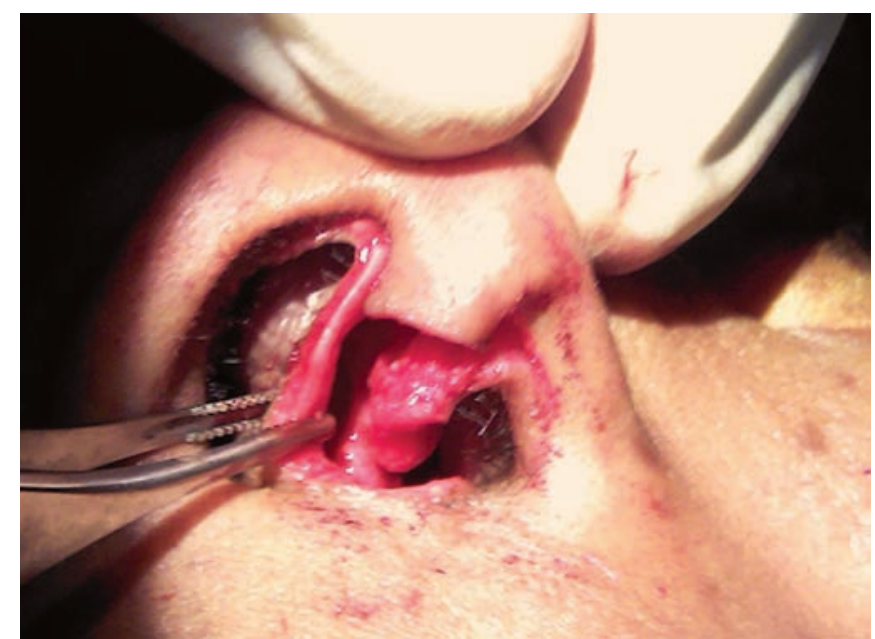

Fig. 5: A CT scan showing left vestibular stenosis

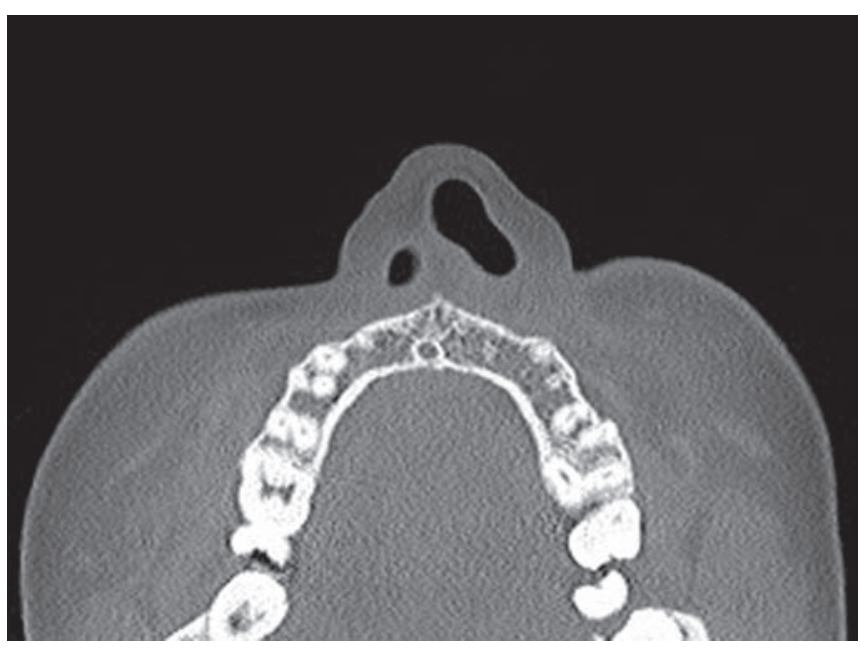

Fig. 4: A CT scan showing underdeveloped left maxillary sinus and cystic swelling over dorsum of the nose

Nasolabial incision is given at the junction of columella and upper lip. Superficial myoaponeurotic flap over the nasal framework is elevated up to nasion (Fig. 6). While elevation of the flap, cystic swelling (measuring about $1 \mathrm{~cm}$ ) over the dorsum was ruptured and collapsed. Upper and lower lateral cartilage, bony nasal framework on the left side of the nose was rudimentary. Septal cartilage harvested with preservation of dorsal and caudal strip of septal cartilage and septal deviation was corrected. ${ }^{3}$ Conchal cartilage harvested and sutured to alar rim to reconstruct external nasal valve., ${ }^{5,6}$ Tip-plasty was done with extended spreader graft, ${ }^{6,7}$ columellar strut, ${ }^{5,6}$ and interdomal sutures. ${ }^{7,8}$

To correct the left nostril stenosis, subnasal rotation flap ${ }^{4}$ harvested from the upper lip at the lower border of right nostril (Fig. 7). Pedicle of flap is aligned in such a way that it should form lower part of columella. Free end of the flap is rotated from right side to the left side, for the widening of the left vestibular opening. Surgical wound 


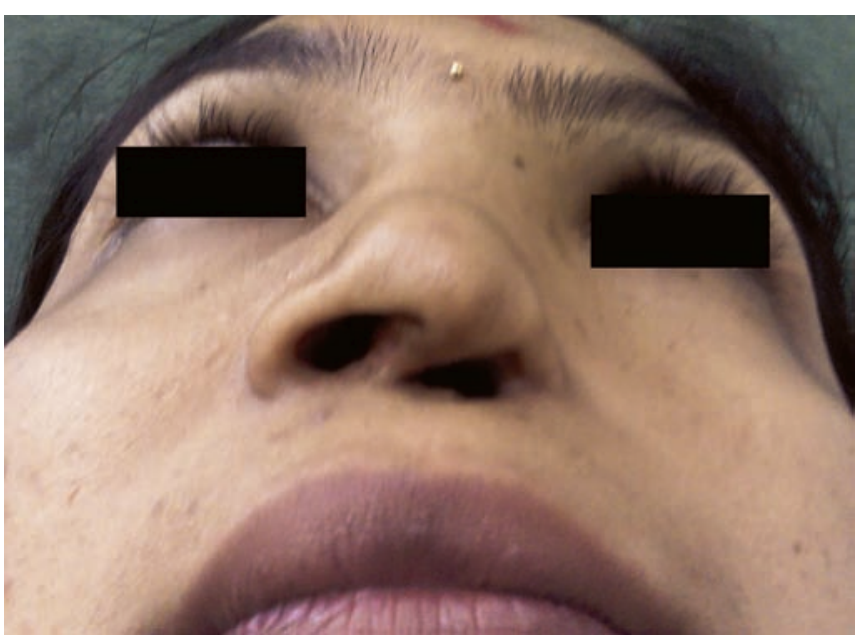

Fig. 7: Intraoperative picture showing subnasal rotation flap

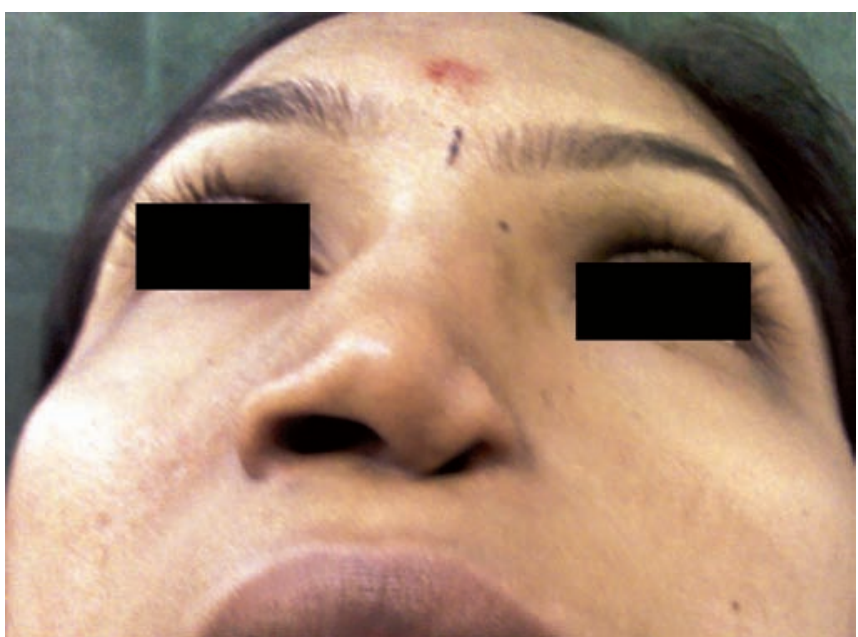

Fig. 9: Postoperative picture, with lateral view showing straight dorsum and nasolabial angle

of flap area is approximated and sutured with 5-0 Prolene. Free end of the subnasal rotation flap is sutured to the floor and columellar part of the left nostril, to increase the surface area of the nostril.

Internal nasal Teflon splint was placed on both sides of the nasal cavity and sutured sandwiching the nasal septum, to prevent adhesions between septum and lateral wall of the nose. And it also helps to clear the nasal crusts during the postoperative period. These splints were removed on 10th postoperative day. Red rubber tube of $3 \mathrm{~cm}$ in length (size: $10 \mathrm{~mm}$ ) is taken and sharp edges are trimmed. And this tube is kept and sutured to newly widen left nostril for 3 weeks. It will prevent the collapse, displacement of the nasal flap, postoperative fibrosis, and restenosis of reconstructed left nostril. External aluminum nasal splint was applied for 10 days. Regular postoperative follow-up and dressing done for wound healing and flap takeup. Sutures were removed on 10th postoperative day. Postoperative picture shows basal view showing reconstructed and wide left nostril

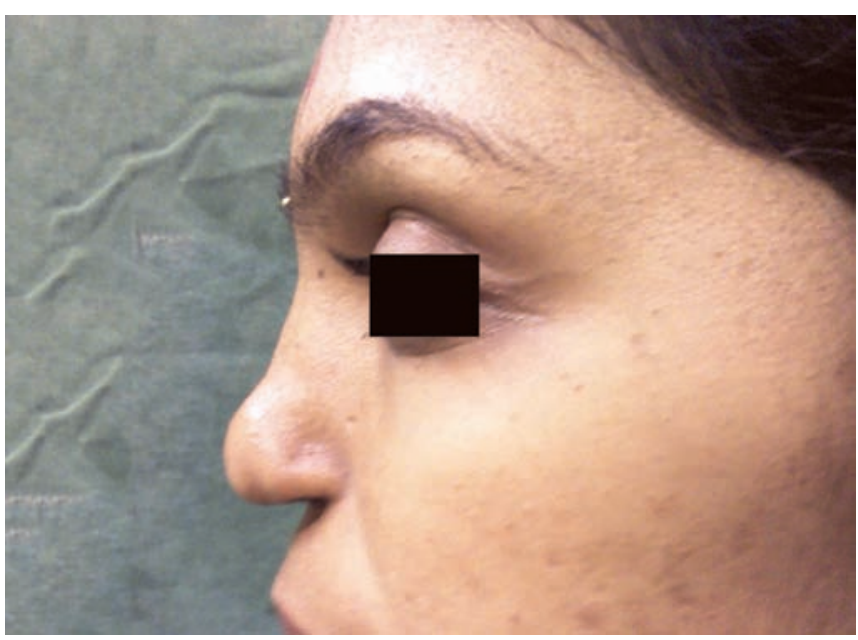

Fig. 8: Postoperative picture, with basal view showing reconstructed and wide left nostril

(Fig. 8) and lateral view showing straight dorsum and nasolabial angle (Fig. 9).

\section{DISCUSSION}

Nasal obstruction can lead to compromised airway and respiratory distress. Our nose is where more than half of this resistance is from. The anterior portion of the nose is where most of the resistance occurs. This area is known as the nasal valve, and it acts as a flow.

The external nasal valve is constructed by the nasal floor, the nasal ala, and by the middle of the nose in the front called the columella. The nasalis muscle expands the external valve area during inhalation. The internal nasal valve is what we normally mean when we are referring to the area called the "nasal valve." The internal nasal valve is where most of the flow resistance is created. It is the area between the nasal septum and the lower border of upper lateral cartilage of the nose. When air comes in the narrow valve, a negative pressure is made and the valve tends to collapse. After inhale is the exhale, where the negative pressure releases and the passage opens to its original position. Nasal valve collapse can be dynamic, fixed, or both.

Nasal hypoplasia ranging from underdevelopment or partial absence of parts to complete arhinia is the most frequently seen nasal anomalies. ${ }^{9}$ Heminasal aplasia, hemi-arhinia, or unilateral aplasia of the nose is a rare congenital malformation in which there is absence of half of the external nose together with a variable degree of abnormality in the internal anatomy of the nose as well as the adjacent facial structures. It will have physiological, psychological, and social impact on the patient. It imposes a major psychological burden to the parents as well.

Failure of the development of both nasal placodes results in complete nasal aplasia or arhinia while failure of one placode leads to heminasal aplasia or hemiarhinia. ${ }^{10}$ Nasal anomalies rarely occur alone and are 
frequently associated with other coexistent craniofacial anomalies. They were classified into two major groups: (1) Total arhinia, with absence of the nose and both olfactory nerves and (2) partial arhinia with presence of at least one nostril and one olfactory tract. Both groups can be seen without (1) or with (2) other craniofacial malformations. ${ }^{11}$ Partial arhinia includes all the range from hypoplasia or absence of only an individual structure to a complete absence of the heminose. Few cases were reported with congenital absence of the columella, with the medial crura of the lower lateral cartilages and their soft-tissue covering were missing, while the remaining septum and other nasal structures were normal. ${ }^{12,13}$ Isolated nasal bone agenesis or hypoplasia has also been reported. ${ }^{14,15}$ The management of heminasal hypoplasia is a surgical challenge because of its rarity and complex anatomy.

In our case, adult lady presented with unilateral nasal obstruction since birth and she wanted to improve the function of nose and also esthetic appearance. We preferred subnasal rotation flap to correct asymmetrical nostril. Red rubber tube (size: $10 \mathrm{~mm}$ ) is kept in the left nostril to avoid restenosis. Internal nasal Teflon splint is placed on both sides of the nasal cavity and sutured sandwiching the nasal septum, to prevent adhesions between septum and lateral wall of the nose. It also helps to stabilize the reconstructed nasal septum and nostril. Regular follow-up visits were made. Examination at the 6th postoperative week showed an adequate nasal passage and her esthetic appearance is also better than earlier.

\section{CONCLUSION}

Heminasal hypoplasia is a rare congenital anomaly and difficult to manage. These patients will have unilateral nasal obstruction and severe facial disfigurement. Computed tomography scan is essential to know the extent of the deformity and to plan the surgery. Subnasal rotation flap ${ }^{4}$ is used to give airway on both sides and better esthetic appearance.

\section{REFERENCES}

1. Mavili E, Akyürek M. Use of upper lip flap for correction of nostril stenosis. Otolaryngol Head Neck Surg 1999 Dec;121(6):840-841.

2. al-Qattan MM, Robertson GA. Acquired nostril stenosis. Ann Plast Surg 1991;27(4):382-386.

3. Caloss R, Sinn DP. Functional rhinoplasty - The assessment and management of obstructive nasal deformities. J Oral Maxillofac Surg 2007 Sep;65(9):80.

4. Jung DH, Lansangan LJ, Choi JM, Jang TY, Lee JJ. Subnasal flap for correction of columellar deformity. Plast Reconstr Surg 2007 Mar;119(3):885-890.

5. Murrell GL. Auricular cartilage grafts and nasal surgery. Laryngoscope 2004 Dec;114(12):2092-2102.

6. Rohrich RJ, Kurkjian TJ, Hoxworth RE, Stephan PJ, Mojallal A. The effect of the columellar strut graft on nasal tip position in primary rhinoplasty. Plast Reconstr Surg 2012 Oct;130(4):926-932.

7. Akkus AM, Eryilmaz E, Guneren E. Comparison of the effects of columellar strut and septal extension grafts for tip support in rhinoplasty. Aesthetic Plast Surg 2013 Aug;37(4): 666-673.

8. Daniel RK. Rhinoplasty: open tip suture techniques: a 25-year experience. Facial Plast Surg 2011 Apr;27(2):213-224.

9. Losee JE, Kirschner RE, Whitaker LA, Bartlett SP. Congenital nasal anomalies: a classification scheme. Plast Reconstr Surg 2004 Feb;113(2):676-689.

10. van Kempen AA, Nabben FA, Hamel BC. Heminasal aplasia: a case report and review of the literature of the last 25 years. Clin Dysmorphol 1997 Apr;6(2):147-152.

11. Cohen D, Goitein KJ. Arhinia revisited. Rhinology 1987 Dec;25(4):237-244.

12. Lewin ML. Congenital absence of the nasal columella. Cleft Palate J 1988 Jan;25(1):58-63.

13. Mavili ME, Akyurek M. Congenital isolated absence of the nasal columella: reconstruction with an internal nasal vestibular skin flap and bilateral labial mucosa flaps. Plast Reconstr Surg 2000 Aug;106(2):393-399.

14. Klinger M, Caviggioli F, Klinger F, Torsello F, Guidarelli P. Isolated congenital absence of the nasal bones and aesthetic surgical correction: managing and case report. Aesthetic Plast Surg 2005 Jul-Aug;29(4):246-249; Discussion 250.

15. Manning KP, Singh SD. Hypoplasia of the nasal bones. J Laryngol Otol 1977 Dec;91(12):1085-1091. 